\title{
Methylation profiling in non-small cell lung cancer: Clinical implications
}

\author{
ALBERTO MORÁN ${ }^{1}$, TAMARA FERNÁNDEZ-MARCELO $^{1}$, JUAN CARRO $^{1}$, CARMEN DE JUAN $^{1}$, \\ IRENE PASCUA ${ }^{1}$, JACQUELINE HEAD ${ }^{1}$, ANA GÓMEZ ${ }^{2}$, FLORENTINO HERNANDO ${ }^{2}$, \\ ANTONIO-JOSÉ TORRES ${ }^{2}$, MANUEL BENITO ${ }^{1}$ and PILAR INIESTA ${ }^{1}$ \\ ${ }^{1}$ Department of Biochemistry and Molecular Biology, Faculty of Pharmacy, Complutense University; \\ ${ }^{2}$ Surgery Service 2, San Carlos Hospital, Health Research Institute \\ from San Carlos Hospital (IdISSC), 28040-Madrid, Spain
}

Received August 30, 2011; Accepted October 7, 2011

DOI: $10.3892 /$ ijo.2011.1253

\begin{abstract}
The aim of this study was to identify a panel of methylation markers that distinguish non-small cell lung cancers (NSCLCs) from normal lung tissues. We also studied the relation of the methylation profile to clinicopathological factors in NSCLC. We collected a series of 46 NSCLC samples and their corresponding control tissues and analyzed them to determine gene methylation status using the Illumina GoldenGate Methylation bead array, which screens up to $1505 \mathrm{CpG}$ sites from 803 different genes. We found that $120 \mathrm{CpG}$ sites, corresponding to 88 genes were hypermethylated in tumor samples and only $17 \mathrm{CpG}$ sites (16 genes) were hypomethylated when compared with controls. Clustering analysis of these 104 genes discriminates almost perfectly between tumors and normal samples. Global hypermethylation was significantly associated with a worse prognosis in stage IIIA NSCLC patients $(\mathrm{P}=0.012)$. Moreover, hypermethylation of the $C A L C A$ and $M M P-2$ genes were statistically associated to a poor clinical evolution of patients, independently of TNM tumor stage $(\mathrm{P}=0.06, \mathrm{RR}=2.64 ; \mathrm{P}=0.04, \mathrm{RR}=2.96$, respectively). However, hypermethylation of RASSF1 turned out to be a protective variable $(\mathrm{P}=0.02 ; \mathrm{RR}=0.53)$. In conclusion, our results could be useful for establishing a gene methylation pattern for the detection and prognosis of NSCLC.
\end{abstract}

\section{Introduction}

Lung cancer is one of the most common cancer malignancies worldwide. According to the World Health Organization, it kills over one million people each year and it is the leading cause

Correspondence to: Dr Pilar Iniesta, Department of Biochemistry and Molecular Biology, Faculty of Pharmacy, Complutense University, 28040-Madrid, Spain

E-mail: insepi@farm.ucm.es

Key words: methylation, non-small cell lung cancer, epigenome, CALCA, MMP-2, RASSF1, prognosis of cancer death in men and second leading cause in women. Clinically, lung cancer is divided into two subtypes, small cell lung cancer (SCLC) and non-small cell lung cancer (NSCLC). SCLC is the most aggressive subtype, and accounts for $10-15 \%$ of all cases. The remaining $85-90 \%$ of cases is classified as NSCLC, which is further subdivided into four histological categories; adenocarcinoma (AD), squamous cell carcinoma (SQ), large cell carcinoma (LC) and 'others' (1). Lung cancer is unique among human solid cancers in that a single environmental factor, tobacco smoke, is believed to promote sequential changes in target cells that lead to carcinogenesis. Recently, progress has been made in elucidating changes involved in the transformation of a normal bronchial epithelium to frank malignancy (2). However, there is not a routine screening method that enables early detection, which is a key factor for decreasing the mortality rate of this cancer. Imaging and cytology analyses have been employed for early detection, but none have been demonstrated to reduce mortality. Thus, the quest for specific molecular markers has become so important in lung cancer.

DNA methylation has emerged as a highly promising biomarker and is being widely studied in every type of cancer. $\mathrm{CpG}$ (cytosine-guanine) islands are short regions of $0.5-4 \mathrm{~kb}$ rich in $\mathrm{CpG}$ content found in almost half of all genes and are frequently promoter-associated $(1,2)$. The addition of a methyl group at the 5-position of the cytosine in a $\mathrm{CpG}$ dinucleotide is a normal process within cells in the mammalian genome and hypermethylation of $\mathrm{CpG}$ islands in promoter regions is a frequent mechanism for gene inactivation. Changes in the pattern of methylation in these regions have been widely described in cancer (3). Although global hypomethylation has been shown in tumors (4), hypermethylation of specific genes is the most frequent and well characterized epigenetic change (5). Therefore, the use of methylation markers to classify different types of cancer and predict patient survival is an interesting field of research.

Disruption of gene expression caused by methylation plays an important role in pathogenesis of NSCLC and it may be relevant as a detection or outcome prediction tool. Several studies have identified aberrant methylation events in different genes in NSCLC $(6,7)$. Our group considers informative the 
study of the methylation status in a large number of genes, in order to establish a panel of relevant genes which are hyper or hypomethylated in a set of NSCLC patients.

\section{Materials and methods}

Patients and tissue samples. Forty-six primary NSCLCs and their corresponding control tissue samples were obtained from patients who underwent surgery between 2000 and 2005, at San Carlos Hospital in Madrid, Spain. Informed consent was obtained from patients prior to investigation and the project was approved by the Ethics Committee of San Carlos Hospital. Samples were snap-frozen in liquid nitrogen at the time of surgery and stored at $-80^{\circ} \mathrm{C}$ until use. No patient had received chemotherapy or radiation therapy before diagnosis. Cryostatsectioned, H\&E-stained samples from each tumor block were examined microscopically, to confirm the presence of $\geq 80 \%$ tumor cells by two independent pathologists. Paired normal tissues, used as controls, were obtained away from the margin of the tumor and microscopically confirmed. Forty-three patients were male and three female and the average age was 64.97 years (range 40-82). Tumors were staged pathologically using the tumor node metastasis (TNM) system (8) and consisted of 20 stage I, 4 stage II and 22 stage IIIA. According to the World Health Organization criteria, 28 tumors were squamous cell carcinomas (SCC) and 18 were adenocarcinomas (AC). The histological classification of tumors was established according to previous criteria (9). Thus, 11 tumors were well differentiated, 24 moderately, and 11 poorly differentiated. The follow-up period for patients was 5 years. The overall median survival of the series was 22.02 months (range 1-60).

DNA methylation profiling. Genomic DNA from fresh frozen tissue was isolated as described by Blin and Stafford (10). DNA methylation profiling using Illumina GoldenGate Methylation bead arrays (Illumina, San Diego, CA) was carried out at the Human Genotyping Unit-CEGEN from the Spanish National Cancer Research Centre (CNIO), Madrid, according to manufacturer's protocol. This array interrogated $1505 \mathrm{CpG}$ loci associated with 807 cancer-related genes. GoldenGate array methylation data are publicly available on the Gene Expression Omnibus archive, accession GSE22290.

Statistical analysis. Illumina BeadStudio Methylation Software was used for data analysis. The methylation level at each $\mathrm{CpG}$ site ( $\beta$-value) was defined as the ratio of the fluorescent signal for methylated (Cy5) allele to the sum of methylated and unmethylated $(\mathrm{Cy} 3)$ alleles $[\beta=(\max (\mathrm{Cy} 5,0)) /(|\mathrm{Cy} 3|+|\mathrm{Cy} 5|+100)]$. $\beta$-value is derived from the 30 replicate methylation measurements and ranged from 0 (completely unmethylated) to 1 (completely methylated). At each locus for each sample the detection P-value was used to determine sample performance. To ensure adequate sample quality, only samples having $>75 \%$ loci with a detection $\mathrm{P}<0.05$ were included for analysis. GoldenGate assay has nine controls which help to monitor each step of the process. Background normalization was carried out for the methylation analysis. The background value is derived by averaging the signals of built-in negative control bead types. Outliers are removed using the median absolute deviation method. The average signal of the negative controls is subtracted from the probe signals as done by Bibikova et al (11). The expected signal for unexpressed targets is equal to zero.

Gene data set was applied to complete-linkage hierarchical clustering analysis using the Spearman Rank correlation method in Cluster v3.0 and the resulting expression map was visualized with JavaTreeview v1.1.5r2. Unsupervised clustering was used to characterize methylation patterns, as performed in previous studies $(12,13)$.

Clinical correlations were established using software SPSS (PASW Statistics 17). HRs for risk of death were estimated from a multivariate Cox proportional hazards model, with adjustments for age, sex, smoking status, tumor grade, tumor stage, and histology. Overall survival in relation to methylation status was evaluated by Kaplan-Meier survival curves and log-rank tests. A p-value $<0.05$ denoted significance.

Validation of microarray by methylation-specific PCR. Methylation status of specific genes was examined by methylationspecific PCR (MSP) (14) after bisulfite treatment. Genomic DNA from samples was subjected to bisulfite modification using the Epitect Bisulfite kit from Qiagen according to manufacturer's instructions. The bisulfite-converted genomic DNA was amplified by PCR using two sets of specific MSP-primers recognizing methylated or unmethylated DNA respectively. PCR conditions and primers for RASSFI were described by Wang et al (15) and for pl6 by Gonzalez-Quevedo et al (16).

\section{Results}

Methylation cluster analysis. DNA from 46 NSCLCs and its 46 control samples were subjected to a study using Illumina GoldenGate bead array to characterize the epigenetic profile. This panel interrogates $1505 \mathrm{CpG}$ loci selected from 807 cancerrelated genes where $28.6 \%$ contain one $\mathrm{CpG}$ site per gene, $57.3 \%$ contain two CpG sites, and $14.1 \%$ have three or more sites. 1044 CpG sites are located within CpG islands, and 461 are located outside the $\mathrm{CpG}$ islands. After analyzing the array, one nontumor sample was taken out from the study, because its signal was too low to obtain reliable results.

Array methylation data were analyzed with unsupervised hierarchical clustering using Spearman Rank correlation for the 1505 CpG sites. As shown in Fig. 1, this cluster analysis helps to distinguish clearly NSCLC samples from non-malignant tissues. Just four paired matched samples (numbers 61, 100, 132 and 133) are not properly classified within its clusters.

In order to determine if a sample shows hypermethylation at one locus, methylation level ( $\beta$ average value) in tumor sample was compared with methylation level at its corresponding nontumor control. When methylation level at tumor sample was at least $50 \%$ higher than methylation level at the corresponding non-tumor control, this sample was classified as "hypermethylated' at that locus. When $\beta$-value at tumor sample was at least $50 \%$ lower than $\beta$-value at non-tumor control, this sample was classified as 'hypomethylated'. Following these criteria, we found that $120 \mathrm{CpG}$ sites, corresponding to 88 genes, were hypermethylated in tumor samples and only $17 \mathrm{CpG}$ sites (16 genes) were hypomethylated when compared with its control (Table I). Remarkably, not all of the $\mathrm{CpG}$ sites analyzed with the microarray are located in a $\mathrm{CpG}$ island. Only those $\mathrm{CpG}$ sites located at a $\mathrm{CpG}$ island are relevant for the expression of 


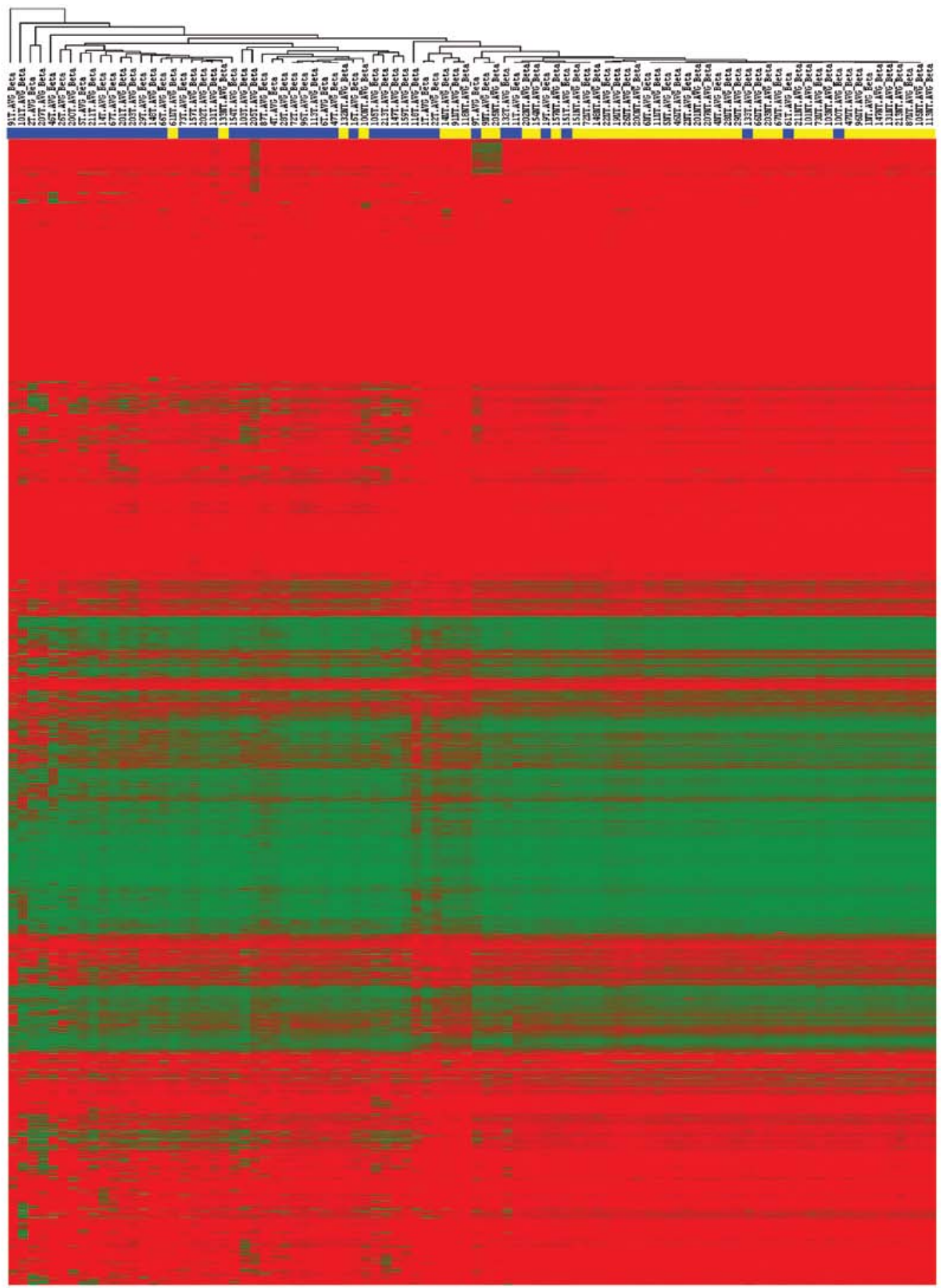

Figure 1. Unsupervised hierarchical clustering of average $\beta$-values in tumor and non-tumor samples, based on Manhattan distance. In the heatmap each column represents a sample (45 tumors, blue, and 45 non-tumors, purple) and each row represents a CpG locus (1505 in 807 genes). Average $\beta$ close to zero, green, means unmethylated and close to one means methylated, red.

the corresponding gene. In Table I those $\mathrm{CpG}$ sites that are not located at $\mathrm{CpG}$ islands are shown in bold. Using the KEGG database (www.genome.jp/kegg/), we identified that most of these genes are involved in proliferation, differentiation or apoptosis pathways. There are genes from the Wnt pathway $(F R Z B, W N T 2$ and SFRPl) and transcription factors (EYA4, HOXA5, HOXA9, HOXA11, HOXB2, HOXB13, IRF5, IRF7, ISL-1 and MYOD1, for example). Adhesion and extracellular matrix molecules also showed an altered methylation pattern $(C D H 13, D C C, O P C M L$ COL18A1, MMP-2, SPARC, TIMP-2, and SPPI). Intriguingly, eight genes, ASCL2, HTR1B, AGTR1, NTSR1, GABRB3, SLIT2,
$P 2 R X 7$ and $G A L R 1$, are involved in signaling pathways related to neuroactive ligand-receptor interaction or axon guidance.

Validation of the microarray by methylation specific PCR (MSP). We validated two $C p G$ sites that showed elevated methylation in NSCLC samples according to Golden Gate microarray using MSP (14). MSP is the most widely used technique to check the methylation status of individual genes. Specific primers to methylated or unmethylated $p 16$ and RASSF 1 were used to amplify DNA samples in separate reactions. We selected randomly 24 patients and checked the methylation status in both tumor and 
Table I. Genes with altered methylation profile. ${ }^{a}$

Hypermethylated genes in NSCLCs

Hypomethylated

genes in NSCLCs

\begin{tabular}{|c|c|c|c|c|c|c|c|}
\hline $\begin{array}{l}\text { Gene } \\
\text { symbol }\end{array}$ & $\begin{array}{c}\text { GeneBank } \\
\text { reference }\end{array}$ & $\begin{array}{c}\text { Gene } \\
\text { symbol }\end{array}$ & $\begin{array}{c}\text { GeneBank } \\
\text { reference }\end{array}$ & $\begin{array}{c}\text { Gene } \\
\text { symbol }\end{array}$ & $\begin{array}{c}\text { GeneBank } \\
\text { reference }\end{array}$ & $\begin{array}{c}\text { Gene } \\
\text { symbol }\end{array}$ & $\begin{array}{l}\text { GeneBank } \\
\text { reference }\end{array}$ \\
\hline$A D C Y A P 1$ & NM_001117 & $H L A-D O A$ & NM_002119 & $N P Y$ & NM_000905 & CASP8 & NM_001228 \\
\hline$A G T R 1$ & NM_000685 & HOXA5 & NM_019102 & NTRK3 & NM_002530 & $C D H 3$ & NM_001793 \\
\hline$A S C L 2$ & NM_005170 & НОХАЯ & NM_002142 & NTSR1 & NM_002531 & IL6 & NM_000600 \\
\hline ATP10A & NM_024490 & HOXA11 & NM_005523 & ONECUT2 & NM_004852 & MEST & NM_002402 \\
\hline$C A L C A$ & NM_001033952 & HOXB2 & NM_002145 & OPCML & NM_002545 & MLLT4 & NM_005936 \\
\hline $\mathrm{CDH} 13$ & NM_001257 & НOХВ 13 & NM_006361 & p16 & NM_058195 & $M U C 1$ & NM_002456 \\
\hline CFTR & NM_000492 & HS3ST2 & NM_006043 & $P 2 R X 7$ & NM_177427 & NEOI & NM_002499 \\
\hline CHFR & NM_018223 & HTRlB & NM_000863 & PENK & NM_006211 & $N R A S$ & NM_002524 \\
\hline$C H G A$ & NM_001275 & $I G F 1 R$ & NM_000875 & PITX2 & NM_000325 & PTPNG & NM_080548 \\
\hline COL18A1 & NM_130444 & $I G F 2$ & NM_000612 & PODXL & NM_001018111 & $R R A S$ & NM_006270 \\
\hline$D A B 2$ & NM_001343 & $I G F 2 A S$ & NM_016412 & PTPRO & NM_002848 & $S F N$ & NM_006142 \\
\hline$D A B 2 I P$ & NM_032552 & $I G F B P 7$ & NM_001553 & $R A R A$ & NM_000964 & SPP1 & NM_000582 \\
\hline$D B C 1$ & NM_014618 & $I G S F 4$ & NM_014333 & RASSF 1 & NM_170712 & $T N F$ & NM_000594 \\
\hline$D C C$ & NM_005215 & $I H H$ & NM_002181 & $S C G B 3 A 1$ & NM_052863 & TNFSF 10 & NM_003810 \\
\hline$D L K 1$ & NM_003836 & $I L 17 R B$ & NM_018725 & SEPT5 & NM_001009939 & $V A M P 8$ & NM_003761 \\
\hline ЕРНA5 & NM_182472 & IL18BP & NM_005699 & SFRP1 & NM_003012 & ZMYND10 & NM_015896 \\
\hline$E P O$ & NM_000799 & IRF5 & NM_032643 & $S L C 22 A 3$ & NM_021977 & & \\
\hline EYA4 & NM_004100 & IRF7 & NM_004029 & SLC5A8 & NM_145913 & & \\
\hline FES & NM_002005 & ISLI & NM_002202 & SLIT2 & NM_004787 & & \\
\hline$F G F 3$ & NM_005247 & $J A K 3$ & NM_000215 & SOX1 & NM_005986 & & \\
\hline FLT3 & NM_004119 & $M A F$ & NM_005360 & SOX17 & NM_022454 & & \\
\hline FLT4 & NM_002020 & MFAP4 & NM_002404 & SPARC & NM_003118 & & \\
\hline$F R Z B$ & NM_001463 & $M M P 2$ & NM_004530 & ST6GAL1 & NM_173216 & & \\
\hline GABRB3 & NM_021912 & $M O S$ & NM_005372 & STAT5A & NM_003152 & & \\
\hline GALRI & NM_001480 & $M T 1 A$ & NM_005946 & $T A L 1$ & NM_003189 & & \\
\hline$G A S 7$ & NM_003644 & MYCL2 & NC_000023 & $T E R T$ & NM_198255 & & \\
\hline GDF10 & NM_004962 & MYOD1 & NM_002478 & TIMP2 & XM_941104 & & \\
\hline$G U C Y 2 D$ & NM_000180 & $N E F L$ & NM_006158 & TPEF & NM_016192 & & \\
\hline \multirow[t]{2}{*}{$H C K$} & NM_002110 & $N P R 2$ & NM_003995 & WNT2 & NM_003391 & & \\
\hline & & & & $W T 1$ & NM_024424 & & \\
\hline
\end{tabular}

${ }^{\mathrm{a}}$ Genes were considered as hypermethylated or hypomethylated after comparing methylation level in tumors to methylation level at the corresponding non-tumor controls.

normal samples. Data obtained from Golden Gate array for $p 16$ and RASSF1 were confirmed in $85.7 \%$ and $95.8 \%$ of the patients (both tumor and normal samples) respectively.

Clinical correlations. Global hypermethylation (more than $20 \%$ of the CpG sites hypermethylated in one tumor sample) did not show statistical correlations with clinical and pathological parameters, such as TNM stage $(\mathrm{P}=0.778)$, size of tumor $(\mathrm{P}=0.479)$, lymph node dissemination $(\mathrm{P}=0.908)$ or histology $(\mathrm{P}=0.541)$. We then search for clinical and pathologic correlations with hypermethylation gene by gene. Statistical analyses of the hypermethylation of single genes did not show significant associations with TNM stage. However, differences in the frequencies of hypermethylation were seen between NSCLC histological subtypes. The frequencies of hypermethylation in twelve genes (CALCA, $\mathrm{P}=0.08, H O X B 2, \mathrm{P}=0.033, I R F 7$, $\mathrm{P}=0.013$, MOS, $\mathrm{P}=0.039$, MT1A, $\mathrm{P}=0.004$, MYCL2, $\mathrm{P}=0.001$, p16, $\mathrm{P}=0.023$, RARA, $\mathrm{P}=0.027$, RASSF1, $\mathrm{P}=0.048$, SEPT5, $\mathrm{P}=0.033, S O X 17, \mathrm{P}=0.039$, and $S P A R C, \mathrm{P}=0.048)$ were significantly higher in squamous cell carcinomas (SCC) compared to adenocarcinomas. Some other genes, such as HOXA5, PTPRO, $S L C 5 A 8$, and ST6GAL1 showed a trend to be more methylated 


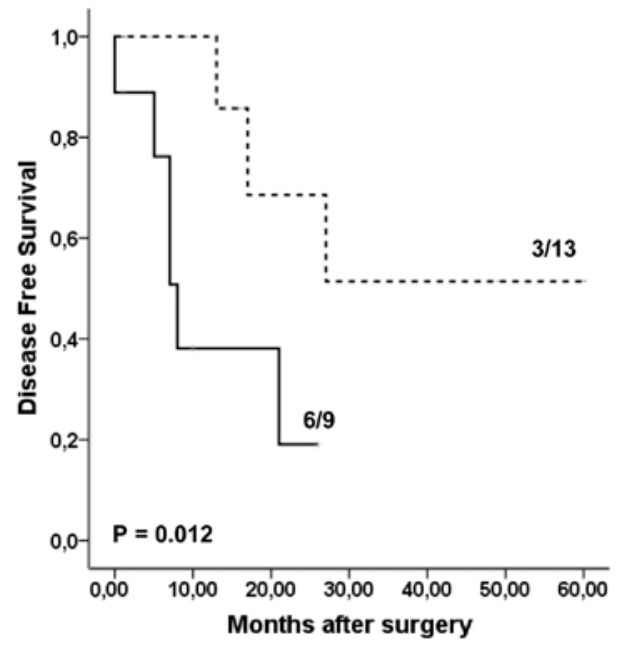

Figure 2. Kaplan-Meier survival curves for TNM stage IIIA NSCLCs showing global hypermethylation. Solid line represents patients with $\mathrm{CpG}$ hypermethylation and broken line represent patients without hypermethylation.

Table II. Cox multivariate analysis for TNM stage and $C A L C A$, $M M P-2$ and $R A S S F 1$ methylation status in non-small cell lung tumors.

\begin{tabular}{|c|c|c|c|}
\hline Variable & $\mathrm{RR}$ & $\begin{array}{l}\text { 95\% Confidence } \\
\text { Interval }\end{array}$ & P-value \\
\hline TNM stage & 1.822 & $1.040-3.192$ & 0.036 \\
\hline$C A L C A$ hypermethylation & 2.643 & $0.953-7.333$ & 0.062 \\
\hline$M M P-2$ hypermethylation & 2.956 & $1.067-8.190$ & 0.037 \\
\hline RASSF 1 hypermethylation & 0.535 & $0.312-0.916$ & 0.023 \\
\hline
\end{tabular}

$\mathrm{RR}$, relative risk.

in SCC compared to adenocarcinomas, but they did not reach statistic significance. Remarkably, no gene showed a trend to be more methylated in adenocarcinomas than in SCC. When we studied the frequencies of hypermethylation and compared them to differentiation grade, we found that well-differentiated tumors showed hypermethylation in IRF5 $(\mathrm{P}=0.039)$ and NPR2 $(\mathrm{P}=0.020)$.

Looking for prognostic factors, we found a tendency to a worse prognosis in those patients whose tumors showed global hypermethylation $(\mathrm{P}=0.065) ; 19$ patients showed hypermethylation in at least $20 \%$ of the $1505 \mathrm{CpG}$ sites studied. This trend toward a worse prognosis was statistically significant in TNM stage IIIA NSCLCs ( $\mathrm{P}=0.012$, Fig. 2). Hypermethylation of genes $C A L C A$ and $M M P-2$ were statistically associated to a worse clinical evolution of patients (Fig. 3A and B, $\mathrm{P}=0.047$ and $\mathrm{P}=0.020$, respectively). However, hypermethylation of RASSF1 turned out to be a protective variable in relation to patient prognosis $(\mathrm{P}=0.010$, Fig. $3 \mathrm{C})$. These results were independent of TNM tumor stage, as demonstrated by a Cox multivariate analysis $(\mathrm{P}=0.06, \mathrm{RR}=2.64 ; \mathrm{P}=0.04, \mathrm{RR}=2.96 ; \mathrm{P}=0.02 ; \mathrm{RR}=0.53$, respectively, shown in Table II).
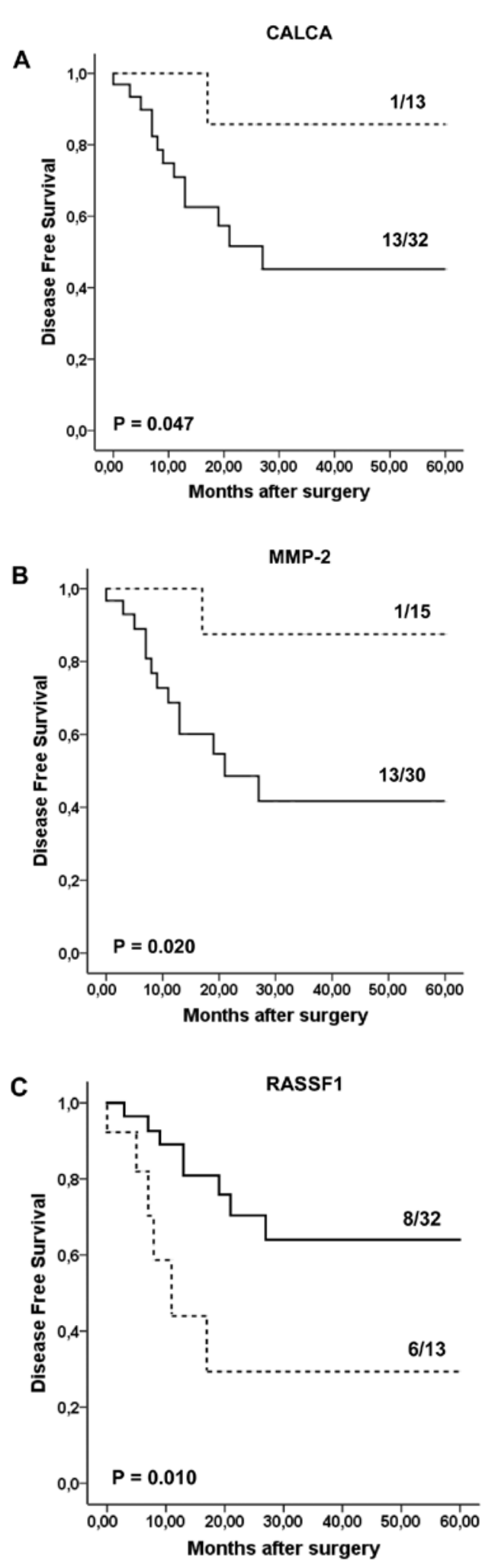

Figure 3. Kaplan-Meier survival curves for CALCA (A), MMP-2 (B) and RASSF1 (C) hypermethylation. Solid lines represent patients whose tumors were hypermethylated and broken lines represent patients without hypermethylation.

We then looked for clinical and pathologic correlations with gene hypomethylation. Global hypomethylation (more than $20 \%$ of the CpG sites hypomethylated) was not statistically associated to a different prognosis in NSCLCs $(\mathrm{P}=0.155)$. However, 


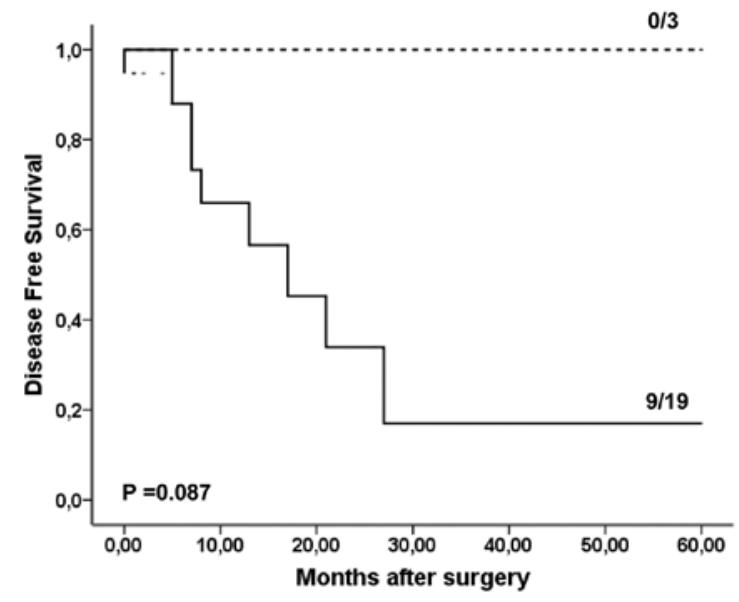

Figure 4. Kaplan-Meier survival curves for hypomethylation. Broken line represents patients with $\mathrm{CpG}$ hypomethylation and solid line represents patients without hypomethylation.

there was a clear trend toward a better prognosis in those patients considered as 'hypomethylated' when compared with non-hypomethylated in TNM stage IIIA NSCLCs $(\mathrm{P}=0.087$, Fig. 4). Statistical analyses of the hypomethylation did not show significant associations with TNM stage or NSCLC histological subtypes. When we studied the frequencies of hypomethylation and compared them to differentiation grade, we found that undifferentiated tumors showed hypomethylation in MLLT4, MUCI and $\operatorname{SFN}(\mathrm{P}=0.025,0.035$ and 0.031 respectively).

\section{Discussion}

The study of the epigenome and its relation to cancer progress is a field of growing interest. In this study, a series of 46 matched NSCLC case-control pairs we assessed the DNA methylation profile for $1505 \mathrm{CpG}$ sites located at 807 cancer-related genes using Illumina GoldenGate bead array. We identified significant differences at $137 \mathrm{CpG}$ sites in 104 independent genes. Of those 137 CpG sites, 120 (88 genes) were hypermethylated in tumor samples versus its corresponding controls and 17 (16 genes), were hypomethylated. In order to validate results obtained from Golden Gate array, we carried-out MSP in a set of patients for RASSF1 and p16. Results were highly concordant with the methylation status determined by the microarray analysis. The overall validity of Illumina Golden-Gate method was already tested by Bibikova et al (11).

It is remarkable that 23 out of these 88 hypermethylated genes have been previously described as hypermethylated in lung cancer: $C A L C A(7), C D H 13$ (17), CHFR (18), DAB2IP (19), DBCl (20), GDFlO (21), HOXA5 (22,23), HOXA9 (24), HOXB13 (25), HTRIB (26), IGFBP7 (7), IGSF4 (27), IRF7 (28), MMP-2 (29), ONECUT2 (25), OPCML (30), pl6 (31,32), PITX2 (33), RASSF1 (34,35), SCGB3A1 (36), SFRP1 (37), SLIT2 (38) and SPARC (31).

It is noteworthy that $R A S S F 1$ is hypermethylated in our samples and this gene has been considered as a tumor suppressor gene (39). Its hypermethylation may cause an inactivation of the gene and it may impair its tumor suppressor gene function. Also remarkable is that our group had previously reported $p 16$ hypermethylation in a set of 98 NSCLCs. Thus, we found that
$36.7 \%$ of samples without p16 expression showed p16 promoter hypermethylation, this fact correlating to adverse patient prognosis (16). Furthermore, it is significant that we found hypermethylated $H O X A 5,9$ and 11 genes, all of them placed in a cluster at the chromosome 7p15.2. Epigenetic inactivation of this $H O X A$ gene cluster has been described in breast and lung cancer (24,40). Likewise, ASCL2, IGF2, IGF2AS and genes are colocalized in chromosome 11p15.5.

Only one out of the sixteen hypomethylated genes has been previously found to suffer hypomethylation in lung cancer: SFN (41). SFN, also called 14-3-3 sigma, has been shown to be upregulated in lung cancer and it is thought to be involved in lung cancer tumorigenesis. Another remarkable result is the hypomethylation of ZMYND10, which has been shown to suffer frequent inactivation by promoter hypermethylation in lung cancers and is considered by some authors as a candidate tumor suppressor gene (42). Further, individualized studies on this gene are needed to clarify its role in cancer.

Unsupervised clustering of the DNA methylation profile of these 104 genes achieves excellent segregation of tumor from non-tumor samples. Thus, this panel may be useful as a screening method in diagnosis. It is important to stress that the use of DNA methylation profiling it is an advantage when comparing with the use of RNA gene expression profile, due to the different stability of RNA and DNA samples. Further studies will be necessary for defining in a more precise way a good panel of genes that could be useful for diagnosis and prognosis.

In the current study we found that methylation of a number of genes was significantly associated with histological type; squamous cell carcinomas was associated with hypermethylation of CALCA, HOXB2, IRF7, MOS, MT1A, MYCL2, p16, RARA, RASSF1, SEPT5, SOX17 and SPARC. We did not find any gene more methylated in adenocarcinomas than in squamous cell carcinomas. However, Hawes et al (43) have recently found that adenocarcinoma was associated with increased methylation of $A P C, C C N D 2, K C N H 5$, and RUNX. Our panel of genes did not include $\mathrm{KCNH} 5$ but the other three genes were included and we could not find any association between its hyper or hypomethylation and any clinical data.

Next, we studied prognostic correlations. First of all there was a clear tendency to a worse prognosis in those patients that showed global hypermethylation and this fact was statistically significant in stage IIIA NSCLCs. Thus, patients with TNM IIIA and hypermethylated tumors showed a clearly worse clinical evolution than those without this molecular event. Therefore, hypermethylation panel in TNM IIIA NSCLCs emerge as a useful molecular tool to predict patient prognosis. Furthermore, the event that we may consider as the 'opposite' of hypermethylation, global hypomethylation, showed clear trend towards a better prognosis in stage IIIA NSCLCs. Therefore, it seems that there is a kind of balance between hyper and hypomethylation that may have prognostic implications. Considering gene by gene methylation, $C A L C A$ and $M M P-2$ were statistically associated to a worse clinical evolution of patients. However, hypermethylation of RASSF1 turned out to be a protective variable in relation to patient prognosis.

$C A L C A$ (calcitonin-related polypeptide $\alpha$ ) is an important regulator of bone calcium metabolism, and it has been found hypermethylated in oligodendrogliomas (44), lymphoblastic 
leukemia (45), and cervical neoplasia (46). Its hypermethylation has also been found in NSCLC (7).

$M M P-2$ is a metalloproteinase that has been shown to be valuable as a prognostic factor in NSCLC (47). MMP-2 is considered as a molecular marker that predicts tumor recurrence and unfavorable outcome in NSCLC (48). Hypermethylation of its promoter has only been described in MCF-7 cells (49). In our set of patients we have found a correlation between $M M P-2$ hypermethylation and a good prognosis. This strengthens its role as a negative prognostic factor.

$R A S S F 1$ is a gene located at $3 \mathrm{p} 21$, a region frequently deleted in human cancer (loss of heterozigosity) that encodes a protein that can bind with NORE1, the novel RAS effector. It is known that RASSF 1 blocks cell cycle progression through a suppression of the c-Jun-NH2-kinase pathway (50) and inhibits cyclin D1 accumulation (51). Altered expression of this gene has been associated with the pathogenesis of a variety of cancers, suggesting a tumor suppressor function of this gene. It is often disrupted by hypermethylation in lung cancer (52). Previously, Tomizawa et al (53) found that epigenetic inactivation of RASSF1 plays an important role in the progression of lung adenocarcinoma, and that RASSF1 hypermethylation correlated with adverse survival in a set of 110 patients with stage I lung adenocarcinoma. Endoh et al (54) showed that RASSF1 methylation was associated with earlier recurrence. However, Choi et al (55) did not consider this gene as a good prognostic marker in a study with 116 cases of NSCLC, although they found that stage I NSCLC patients with RASSF1 inactivation have a more adverse overall survival rate than stage I NSCLC patients without RASSF1 inactivation. We agree with Buckingham et al (35), that epigenetic RASSF1 inactivation may be a good prognostic factor. Our results are supported by the fact that RASSF1 inactivation is related to cell division defects and induces premature $A P C$ activation, thereby resulting in acceleration of mitotic cyclin degradation and mitotic progression as well as induction of mitotic abnormalities (56).

In conclusion, in this study we have established a panel of 104 genes whose methylation status is altered in NSCLCs when compared to its corresponding non-tumor controls. These genes discriminate almost perfectly tumor samples from non-tumor samples. Moreover, global hypermethylation confers a poor prognosis to NSCLC patients, especially in patients affected by TNM stage IIIA tumors. We have found three genes whose hypermethylation may be a useful tool as a prognostic factor: $C A L C A$ and $M M P-2$ hypermethylation confers poor clinical evolution and RASSF1 hypermethylation confers a good prognosis to NSCLC patients.

\section{Acknowledgements}

This study was supported by grants from Ministerio de Sanidad y Consumo (FIS PI080033), Fundación de Investigación Médica Mutua Madrileña, and RTICC RD06/0020/0021.

\section{References}

1. Anglim PP, Alonzo TA and Laird-Offringa IA: DNA methylation-based biomarkers for early detection of non-small cell lung cancer: an update. Mol Cancer 7: 81, 2008.

2. Alberg AJ, Brock MV and Samet JM: Epidemiology of lung cancer: looking to the future. J Clin Oncol 23: 3175-3185, 2005.
3. Esteller M, Fraga MF, Paz MF, et al: Cancer epigenetics and methylation. Science 297: 1807-1808, 2002.

4. Das PM and Singal R: DNA methylation and cancer. J Clin Oncol 22: 4632-4642, 2004.

5. Jones PA and Baylin SB: The fundamental role of epigenetic events in cancer. Nat Rev Genet 3: 415-428, 2002.

6. Toyooka S, Toyooka KO, Maruyama R, et al: DNA methylation profiles of lung tumors. Mol Cancer Ther 1: 61-67, 2001.

7. Wang Y, Zhang D, Zheng W, Luo J, Bai Y and Lu Z: Multiple gene methylation of non small cell lung cancers evaluated with 3-dimensional microarray. Cancer 112: 1325-1336, 2008.

8. Mountain CF: A new international staging system for lung cancer. Chest 89 (Suppl. 4): S225-S233, 1986.

9. Sobin L: The World Health Organization's histological classification of lung tumours: a comparison of the first and second editions. Cancer Detect Prev 5: 391-406, 1982.

10. Blin N and Stafford DW: A general method for isolation of high molecular weight DNA from eukaryotes. Nucleic Acids Res 3: 2303-2308, 1976

11. Bibikova M, Lin Z, Zhou L, et al: High-throughput DNA methylation profiling using universal bead arrays. Genome Res 16: 383-393, 2006.

12. Byun HM, Siegmund KD, Pan F, Weisenberger DJ, Kanel G, Laird PW and Yang AS: Epigenetic profiling of somatic tissues from human autopsy specimens identifies tissue- and individualspecific DNA methylation patterns. Hum Mol Genet 18: 4808-4817, 2009.

13. Shen L, Toyota M, Kondo Y, et al: Integrated genetic and epigenetic analysis identifies three different subclasses of colon cancer. Proc Natl Acad Sci USA 104: 18654-18659, 2007.

14. Herman JG, Graff JR, Myöhänen S, Nelkin BD and Baylin SB: Methylation-specific PCR: a novel PCR assay for methylation status of CpG islands. Proc Natl Acad Sci USA 93: 9821-9826, 1996.

15. Wang T, Liu H, Chen Y, Liu W, Yu J and Wu G: Methylation associated inactivation of RASSF1A and its synergistic effect with activated K-Ras in nasopharyngeal carcinoma. J Exp Clin Cancer Res 28: 160, doi: 10.1186/1756-9966-28-160, 2009.

16. González-Quevedo R, García-Aranda C, Morán A, et al: Differential impact of p16 inactivation by promoter methylation in non-small cell lung and colorectal cancer: clinical implications. Int J Oncol 24: 349-355, 2004.

17. Saito K, Kawakami K, Matsumoto I, Oda M, Watanabe G and Minamoto G: Long interspersed nuclear element 1 hypomethylation is a marker of poor prognosis in stage IA non-small cell lung cancer. Clin Cancer Res 16: 2418-2426, 2010.

18. Mizuno K, Osada H, Konishi H, et al: Aberrant hypermethylation of the CHFR prophase checkpoint gene in human lung cancers. Oncogene 21: 2328-2333, 2002.

19. Yano M, Toyooka S, Tsukuda K, et al: Aberrant promoter methylation of human DAB2 interactive protein (hDAB2IP) gene in lung cancers. Int J Cancer 113: 59-66, 2005.

20. Izumi H, Inoue J, Yokoi S, et al: Frequent silencing of DBC1 is by genetic or epigenetic mechanisms in non-small cell lung cancers. Hum Mol Genet 14: 997-1007, 2005.

21. Dai Z, Popkie AP, Zhu WG, et al: Bone morphogenetic protein 3B silencing in non-small-cell lung cancer. Oncogene 23: 3521-3529, 2004.

22. Shiraishi M, Sekiguchi A,Terry MJ, et al: A comprehensive catalog of $\mathrm{CpG}$ islands methylated in human lung adenocarcinomas for the identification of tumor suppressor genes. Oncogene 21: 3804-3813, 2002.

23. Kim DS, Kim MJ, Lee JY, et al: Epigenetic inactivation of Homeobox A5 gene in nonsmall cell lung cancer and its relationship with clinicopathological features. Mol Carcinog 48: 1109-1115, 2009.

24. Rauch T, Wang Z, Zhang X, et al: Homeobox gene methylation in lung cancer studied by genome-wide analysis with a microarraybased methylated CpG island recovery assay. Proc Natl Acad Sci USA 104: 5527-5532, 2007.

25. Rauch T, Li H, Wu X and Pfeifer GP: MIRA-assisted microarray analysis, a new technology for the determination of DNA methylation patterns, identifies frequent methylation of homeodomain-containing genes in lung cancer cells. Cancer Res 66: 7939-7947, 2006.

26. Takai D, Yagi Y, Wakazono K, Ohishi N, Morita Y, Sugimura T and Ushijima T: Silencing of HTR1B and reduced expression of EDN1 in human lung cancers, revealed by methylation-sensitive representational difference analysis. Oncogene 20: 7505-7513, 2001. 
27. Kikuchi S, Yamada D, Fukami T, et al: Hypermethylation of the TSLC1/IGSF4 promoter is associated with tobacco smoking and a poor prognosis in primary nonsmall cell lung carcinoma. Cancer 106: 1751-1758, 2006.

28. Fukasawa M, Kimura M, Morita S, et al: Microarray analysis of promoter methylation in lung cancers. J Hum Genet 51: 368-374, 2006.

29. Tessema M, Yu YY, Stidley CA, Machida EO, Schuebel KE, Baylin SB and Belinsky SA: Concomitant promoter methylation of multiple genes in lung adenocarcinomas from current, former and never smokers. Carcinogenesis 30: 1132-1138, 2009.

30. Tsou JA, Galler JS, Siegmund KD, et al: Identification of a pane of sensitive and specific DNA methylation markers for lung adenocarcinoma. Mol Cancer 6: 70, 2007.

31. Yoshino M, Suzuki M, Tian L, et al: Promoter hypermethylation of the p16 and Wif-1 genes as an independent prognostic marker in stage IA non-small cell lung cancers. Int J Oncol 35: 1201-1209, 2009.

32. De Jong WK, Verpooten GF, Kramer H, Louwagie J and Groen HJ: Promoter methylation primarily occurs in tumor cells of patients with non-small cell lung cancer. Anticancer Res 29: 363-369, 2009.

33. Anglim PP, Galler JS, Koss MN, et al: Identification of a panel of sensitive and specific DNA methylation markers for squamous cell lung cancer. Mol Cancer 7: 62, 2008.

34. Vaissière T, Hung RJ, Zaridze $\mathrm{D}$, et al: Quantitative analysis of DNA methylation profiles in lung cancer identifies aberrant DNA methylation of specific genes and its association with gender and cancer risk factors. Cancer Res 69: 243-252, 2009.

35. Buckingham L, Penfield Faber L, Kim A, et al: PTEN, RASSF1 and DAPK site-specific hypermethylation and outcome in surgically treated stage I and II nonsmall cell lung cancer patients. Int J Cancer 126: 1630-1639, 2010.

36. Shigematsu H, Suzuki M, Takahashi T, et al: Aberrant methylation of HIN-1 (high in normal-1) is a frequent event in many human malignancies. Int J Cancer 113: 600-604, 2005

37. Licchesi JD, Westra WH, Hooker CM, Machida EO, Baylin SB and Herman JG: Epigenetic alteration of Wnt pathway antagonists in progressive glandular neoplasia of the lung. Carcinogenesis 29: 895-904, 2008

38. Dammann R, Strunnikova M, Schagdarsurengin U, et al: $\mathrm{CpG}$ island methylation and expression of tumour-associated genes in lung carcinoma. Eur J Cancer 41: 1223-1236, 2005.

39. Hesson LB, Cooper WN and Latif F: Evaluation of the 3p21.3 tumour-suppressor gene cluster. Oncogene 26: 7283-7301, 2007.

40. Novak P, Jensen T, Oshiro MM, et al: Epigenetic inactivation of the HOXA gene cluster in breast cancer. Cancer Res 66: 10664-10670, 2006.

41. Osada H, Tatematsu Y and Yatabe Y: Frequent and histological type-specific inactivation of 14-3-3sigma in human lung cancers. Oncogene 21: 2418-2424, 2002.
42. Ito M, Ito G, Kondo M, et al: Frequent inactivation of RASSF1A BLU, and SEMA3B on 3p21.3 by promoter hypermethylation and allele loss in non-small cell lung cancer. Cancer Lett 225: 131-139, 2005.

43. Hawes SE, Stern JE, Feng Q, et al: DNA hypermethylation of tumors from non-small cell lung cancer (NSCLC) patients is associated with gender and histologic type. Lung Cancer 69: 172-179, 2010.

44. Uhlmann K, Rohde K, Zeller C, et al: Distinct methylation profiles of glioma subtypes. Int J Cancer 106: 52-59, 2003.

45. Paixão VA, Vidal DO, Caballero OL, Vettore AL, Tone LG, Ribeiro KB and Lopes LF: Hypermethylation of $\mathrm{CpG}$ island in the promoter region of CALCA in acute lymphoblastic leukemia with central nervous system (CNS) infiltration correlates with poorer prognosis. Leuk Res 30: 891-894, 2006.

46. Wisman GB, Nijhuis ER, Hoque MO, et al: Assessment of gene promoter hypermethylation for detection of cervical neoplasia. Int J Cancer 119: 1908-1914, 2006.

47. Qian Q, Wang Q, Zhan P, Peng L, Wei SZ, Shi Y and Song Y: The role of matrix metalloproteinase 2 on the survival of patients with non-small cell lung cancer: a systematic review with metaanalysis. Cancer Invest 28: 661-669, 2010.

48. Leinonen T, Pirinen R, Böhm J, Johansson R and Kosma VM: Increased expression of matrix metalloproteinase-2 (MMP-2) predicts tumour recurrence and unfavourable outcome in non-small cell lung cancer. Histol Histopathol 23: 693-700, 2008.

49. Chernov AV, Sounni NE, Remacle AG and Strongin AY: Epigenetic control of the invasion-promoting MT1-MMP/MMP-2/TIMP-2 axis in cancer cells. J Biol Chem 284: 12727-12734, 2009.

50. Whang YM, Kim YH, Kim JS and Yoo YD: RASSF1A suppresses the c-Jun-NH2-kinase pathway and inhibits cell cycle progression. Cancer Res 65: 3682-3690, 2005.

51. Shivakumar L, Minna J, Sakamaki T, Pestell R and White MA: The RASSF1A tumor suppressor blocks cell cycle progression and inhibits cyclin D1 accumulation. Mol Cell Biol 22: 4309-4318, 2002.

52. Burbee DG, Forgacs E, Zöchbauer-Müller S, et al: Epigenetic inactivation of RASSF1A in lung and breast cancers and malignant phenotype suppression. J Natl Cancer Inst 93: 691-699, 2001.

53. Tomizawa Y, Kohno T, Kondo H, et al: Clinicopathological significance of epigenetic inactivation of RASSF1A at 3p21.3 in stage I lung adenocarcinoma. Clin Cancer Res 8: 2362-2368, 2002 .

54. Endoh H, Yatabe Y, Shimizu S, Tajima K, Kuwano H, Takahashi T and Mitsudomi T: RASSF1A gene inactivation in non-small cell lung cancer and its clinical implication. Int J Cancer 106: 45-51, 2003.

55. Choi N, Son DS, Song I, et al: RASSF1A is not appropriate as an early detection marker or a prognostic marker for non-small cell lung cancer. Int J Cancer 115: 575-581, 2005.

56. Song MS and Lim DS: Control of APC-Cde20 by the tumor suppressor RASSF1A. Cell Cycle 3: 574-576, 2004. 